\title{
A Hardware-in-Loop Simulation System for Fractionated Spacecraft Cluster
}

\author{
An Meiyan ${ }^{1,2}$, Wang Zhaokui ${ }^{1}$ and Zhang Yulin ${ }^{1}$ \\ 1. School of Aerospace Engineering, Tsinghua University, Beijing 100084, China \\ 2. China Astronaut Research and Training Center, Beijing 100093, China
}

Received: January 26, 2014 / Accepted: July 20, 2014 / Published: January 30, 2015.

\begin{abstract}
FSC (Fractionated Spacecraft Cluster) is a kind of loosely distributed space system which is comprised of multiple physically independent spacecrafts orbiting closely and interactively communicating via wireless network. Spaceborne ad-hoc network, as the physical infrastructure for information exchanging, is one of the enabling technologies of FSC. The demonstration of FSC flying supported by ad-hoc network is worth for the sake of proving the rationality of FSC and seeking technological improvements. Considering this, a NNP (Network Node Prototype) for spaceborne ad-hoc network is developed in this paper, which transmits the information required by the FSC cooperation. Four NNPs are then built up and collaborated into a hardware-in-loop simulation system, in which a typical loose satellite cluster flying mission was performed. The simulation results showed that the NNPs can support the inter-satellite communication for satellite cluster flying.
\end{abstract}

Key words: Fractionated spacecraft cluster, spaceborne ad-hoc network, prototype, hardware-in-loop simulation.

\section{Introduction}

FSC (Fractionated Spacecraft Cluster) is a hot topic in the field of distributed satellite system recently. Usually, a FSC is comprised of many physically independent spacecrafts which orbit closely and interactively communicate with each other via wireless network. It changes the concept of traditional monolithic spacecraft. In the traditional approaches, spacecrafts are allocated to each mission that is associated with high risks and costs and long cycles. While, in a FSC, a set of reconfigurable spacecraft modules on orbit forms a spacecraft system in space, instead of being integrated into a complete spacecraft in the manufacture plant. Meanwhile, the reconfigurable module could be high-volume manufactured, launched separately and interacted freely. Those properties endow FSC with many advantages such as capability of system reconstruction,

Corresponding author: Wang Zhaokui, ph.D., research field: spacecraft cluster control and application. E-mail: wangzk@tsinghua.edu.cn. flexibility of mission accomplishment, replaceable character of specific unit, and robustness of malfunction [1, 2].

FSC can be applied to Earth observation, space exploration, and other space fields. Refs. [1, 3] presented the Magnetic Nano Probe Swarm mission utilizing a constellation of several swarms of nano satellites in order to acquire simultaneous measurements of the geomagnetic field resolving the local field gradients. The space segment is comprised of up to four swarms each consisting of up to six nano satellites and one mother satellite. The ANTS (Autonomous Nano-Technology Swarm), a fractionated spacecraft cluster mission proposed by NASA, involves a swarm of autonomous pico-class spacecrafts that will be used to explore the asteroid belt [4-6]. Another asteroid explore project APIES (Asteroid Population Investigation \& Exploration Swarm) is a "swarm" mission developed by EADS (European Aeronautic Defense and Space Company), based on the utilization of 20 spacecrafts including 
one HIVE (Hub and Interplanetary VEhicle) and 19 BEEs (BElt Explorers) working cooperatively to finish the overall mission objectives [7]. The DARPA (Defense Advanced Research Projects Agency) has commenced a space system plan F6 — short for Future Fast, Flexible, Fractionated, Free-Flying Spacecraft, that provides a future-oriented spacecraft architecture, which disassembles the traditional monolithic spacecraft into a combination of separate modules. Each separate module can be mass manufactured and launched separately. Those modules which stay in a cluster flight formation on orbit, exchange information through wireless communication, and transfer energy through wireless transceiver, constituting a fully functional virtual cluster spacecraft. F6 plan can be seen as a true primary fractionated spacecraft cluster [8]. According to some reports, DARPA canceled F6 plan in 2013, while, in the same reports, it was addressed that "F6 might have been killed off but its idea are still very much alive".

Information exchanging among the fractionated module is one of the important constituents of F6 Plan. Hence, the information exchanging is the enabling technology for the effectively operation of FSC. To fulfill the system reconstruction, flexibility of mission accomplishment, replaceable character of specific unit, and robustness of malfunction, it is necessary for the information exchanging network to be flexible, reconstructive and robust. The wireless ad-hoc network is an infrastructure-less mobile network that has no fixed routers, where all nodes can be connected dynamically in arbitrary manners. All nodes of the network function as routers which discover and maintain routes to other nodes in the network, and function as terminals at the same time [9-11]. These features of ad-hoc network make it very suitable for spacecraft cluster.

The application of ad-hoc network in space is a widely investigated subject, such as Ref. [12] which proposed a wireless sensor network for in situ probing of lunar water/ice and Ref. [13] which utilized ad-hoc wireless sensor network to explore the Solar System. The data transmitting in those networks were the payload data provided by the sensor network nodes. In this paper, the information for FSC cooperation was transmitted with the help of ad-hoc wireless network. Not strictly speaking, the coordination among the spacecraft in FSC was achieved under the support of the wireless network.

In this paper, the authors built a wireless ad-hoc network node, which uses the shelf chip Jennic5148 as its hardware. Based on the nodes as the spacecraft cluster network node prototype, a hardware-in-loop simulation system for fractionated spacecraft cluster was established. The objective of the system is to build a demonstration platform for analysis and simulation of the cluster operation, individual spacecraft control, information exchange, and so on. Finally, a task simulation of loose flight formation including four spacecrafts was performed on this platform.

\section{The Wireless Ad-hoc Network Node Prototype}

The wireless ad-hoc network node has two communicate modes, i.e., peer-to-peer and broadcast. In peer-to-peer mode, a message from source node will be transmitted to the destination node via intermediate nodes chosen according to the route protocol. While in broadcast mode, a node broadcasts message and its neighbor nodes can receive that message at the same time. The wireless ad-hoc network node prototype is composed of communication hardware and embedded control software.

\subsection{Development of the Communication Hardware}

The communication hardware (Fig. 1) includes wireless transceiver chip and interface expansion board. The wireless transceiver chip, as the core part of the hardware, receives radio signals, processes the received signals and transmits the generated signals. 


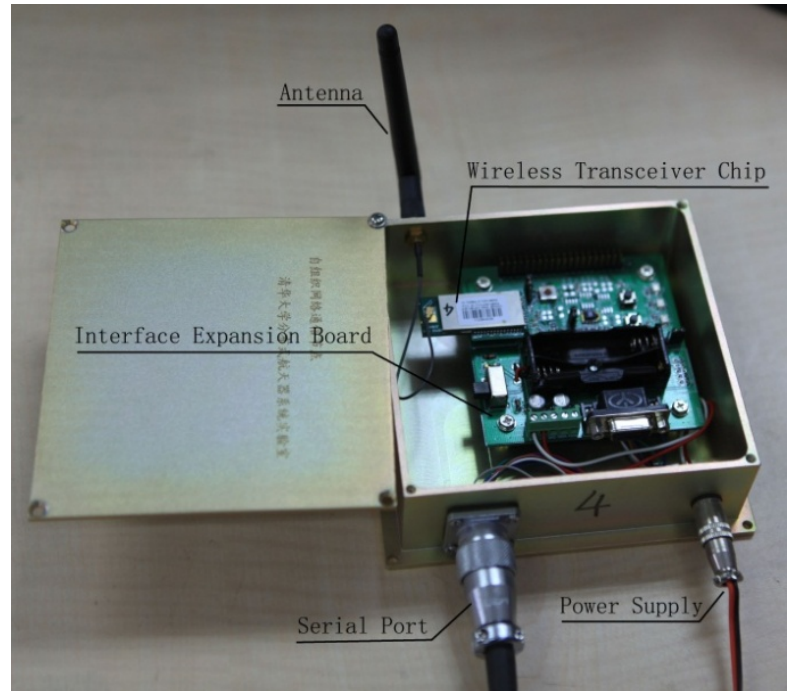

Fig. 1 Wireless ad-hoc network node prototype.

The function of the interface expansion board is to provide power and interactive interface for the wireless transceiver chip.

In order to simplify the complexity of the design process, Jennic 5148 module, a commercial on-shelf product, was selected as the wireless transceiver chip. Jennic5148 module is a high performance surface mount RF (Radio Frequency) chip with ultra-low power requirement and large memory. It has a 32-bit RISC CPU allowing software to be run on chip, a 128 $\mathrm{kB}$ ROM to store program code, a $128 \mathrm{kB}$ RAM to store system data, three system timers, and two UARTs. The RF components are operated in the
2.4 GHz frequency band which is internationally free radio frequency band.

The interface expansion board is comprised of a group of switches, buttons, serial interface, external power connector, etc. It has two operation modes including the development and transceiver modes. Under development mode, it is used to inject the compiled binary embedded control program file into the wireless transceiver chip through the serial port. Under transceiver mode, it allows Jennic5148 module to send and receive data in accordance with the control program.

\subsection{Design of the Embedded Control Software}

The embedded control software, which was developed based on the standard IEEE 802.15.4, has three primary functions including node initialization, data transmitting control, and data receiving control. Node initialization function sets all parameters involving node identity, communication channel, and so on. Data transmission and control functions triggered by interrupt timer periodically, checks whether the sending data buffer has data to be transmitted and sends the data. Receiving control function, enclosed in an overall loop, processes the received data. The flowchart of embedded software is shown in Fig. 2.

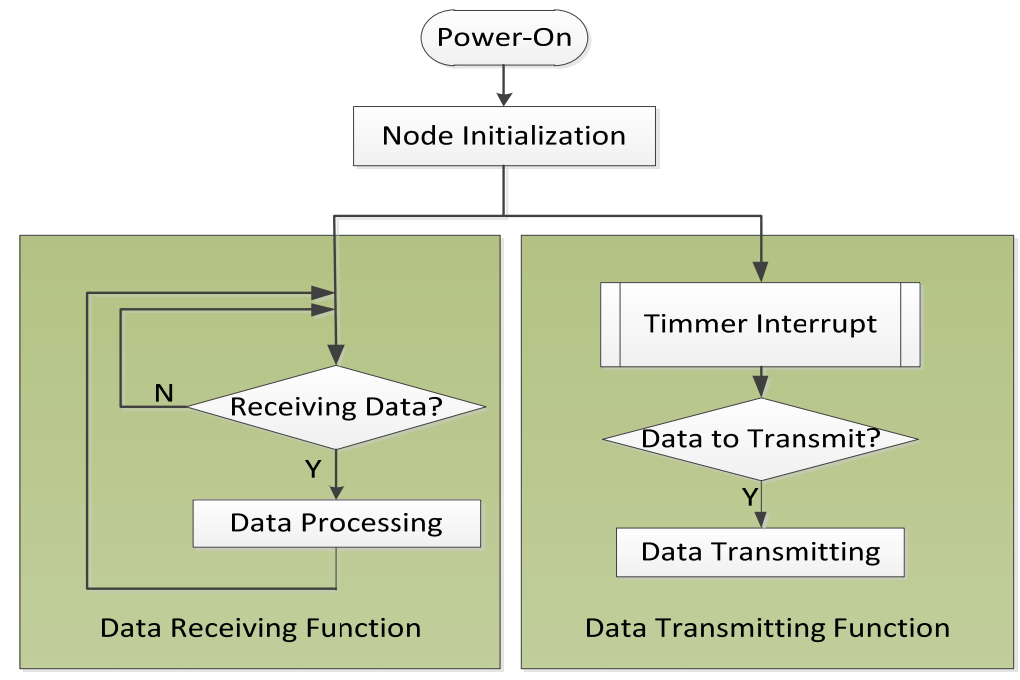

Fig. 2 Flowchart of the embedded software. 
Especially, a frame matching approach was adopted to discriminate the repeated data frame, which might be caused by the multipath effect or other instable factors. Frame matching approach was illustrated in Fig. 3. The transmitting node $i$ counts the frames that was transmitted to receiving node $j$. When node $i$ transmits a frame to node $j$, it enclosed the transmitting sequence number $j$ in the frame head. The receiving node sets a received counter for each node, for example the received counter for node $i$ is $i$ in Fig. 3. When it receives a data frame from node $i$, it firstly checks the frame head and picks up the transmitting sequence number $j$. Then it compares the quantity of the transmitting sequence number $j$ and the received counter $i$. If $i$ is greater than $j$, the receiving frame must be a repeated frame and must be discarded. If $i$ is smaller than $j$, it could be concluded that some data frames must have been missed. To acquire the missed data frames, the receiving node might send retransmission request to the sending node. If those data frame are not needed to retrieve, the receiving node $j$ assigns the value of $j+1$ to the receiving counter $i$ to ensure that the next data frame could be received correctly. If $i$ is equal to $j$, the receiving data frame is correct. Using the frame matching approach, the receiving node could determine whether the receiving data frame is in correct sequence, or discard the repeated frame and retrieve the missed frame.

\section{The Overall Structure of the Simulation System}

Based on four network node prototypes, a hardware-in-loop simulation system for FSC is established. Besides the network node prototype, the simulation system also contains an OCS (On-board Computer Simulator) and a DS (Dynamical Simulator). Fig. 4 shows a photo of the hardware-in-loop simulation system for FSC. Fig. 5 illustrates the architecture of the simulation system, which could be divided into three parts, the IEP (Information Exchanging Platform), SP (Spacecraft Prototype) and dynamical simulation environment. The IEP is constructed by four network node prototypes and can provide the information flow for OCS. The OCS simulator is used to produce the control commands according to the predefined control algorithm and the information received from the IEP. The DS, connected to the on-board computer simulator via serial port, calculates the orbital data for SP. It receives the control commands from the OCS, evolving the next orbital data, and sent the orbital data back to the OCS.

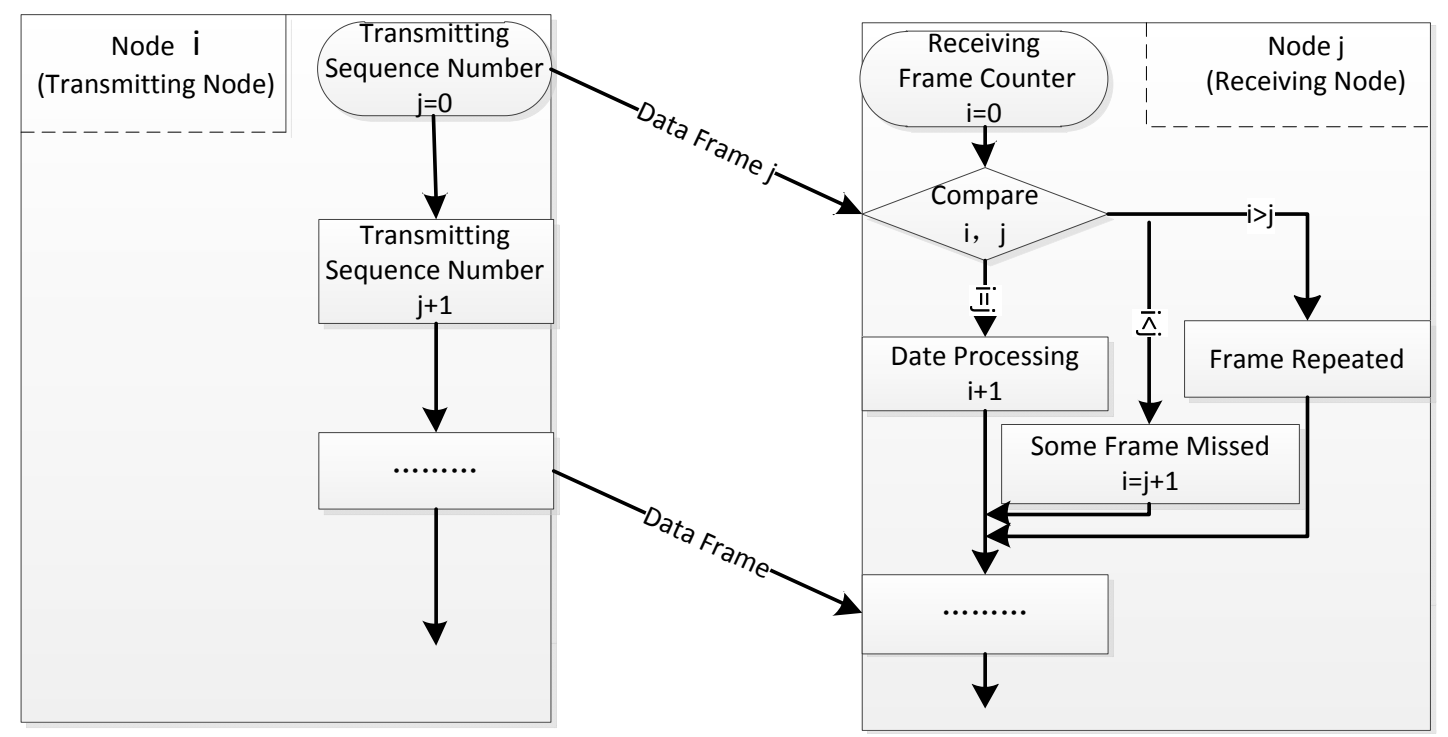

Fig. 3 Frame matching approach. 

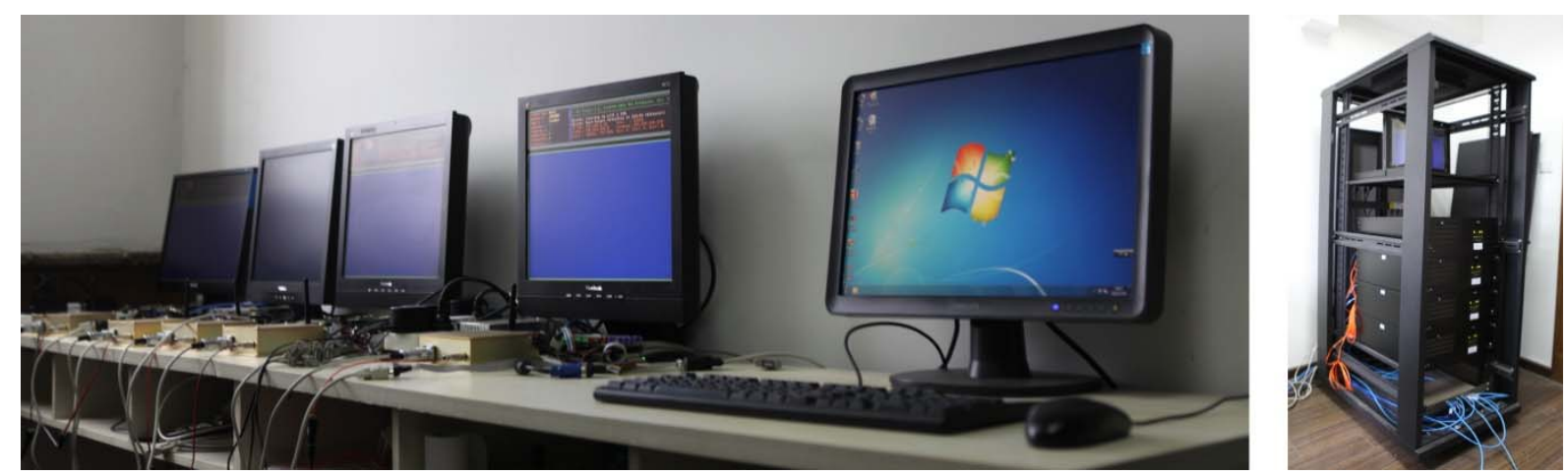

Fig. 4 Hardware-in-loop simulation system for FSC.

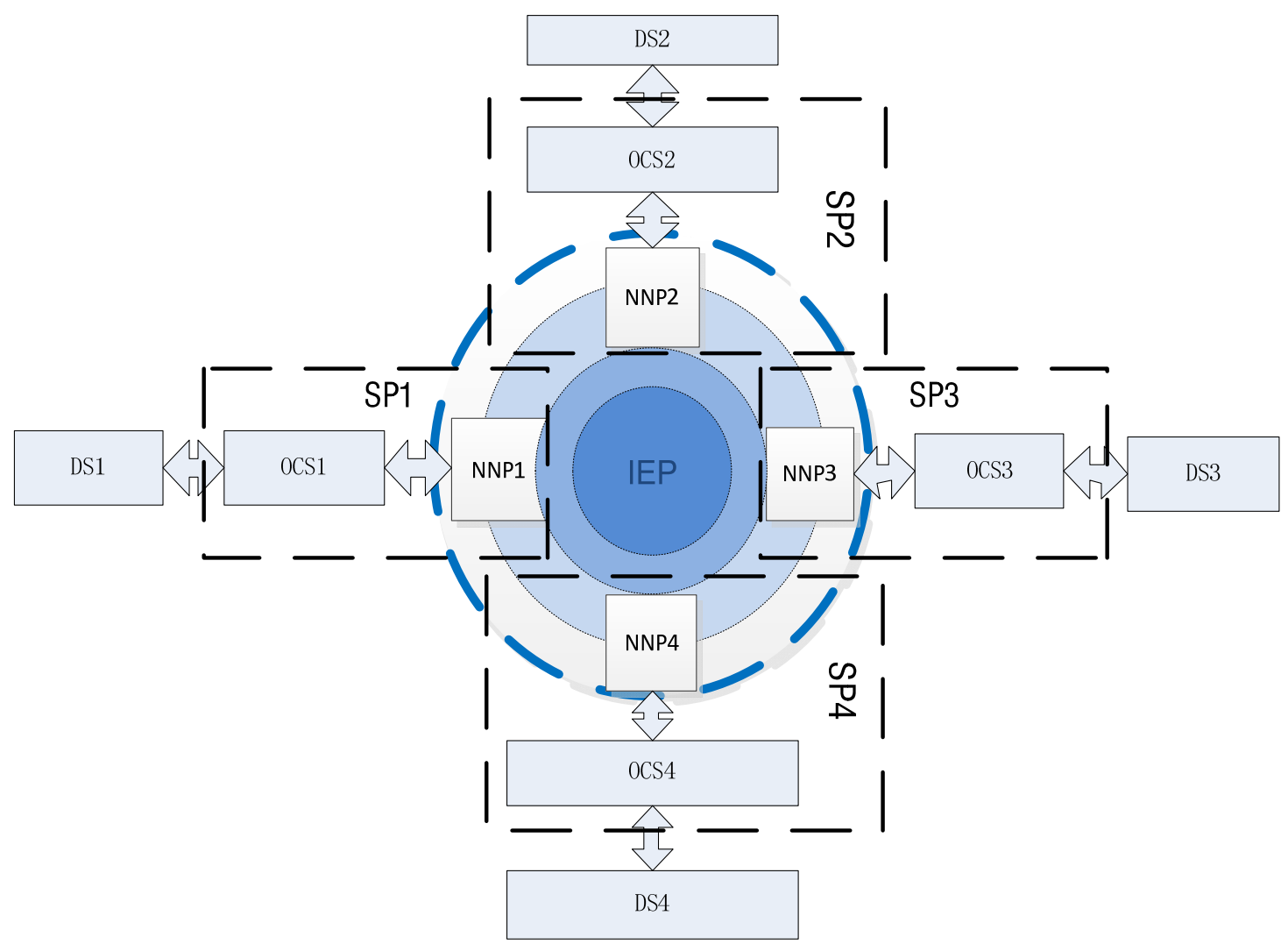

Fig. 5 Architecture of the hardware-in-loop simulation system for FSC.

The simulation system uses the xPC-Target, a Matlab toolkit for real-time signal acquisition and control application, to develop the software of the simulation environment. xPC-Target runs in a host computer connected with the four OCSs and the four DSs via a network switch. The programs for the OCS and the DS are developed in the host computer firstly. Especially, the programs for the on-board computer were specially developed for specialized tasks. While the programs for the DS could be universal for different tasks. Those programs must be downloaded to the OCS and the DS beforehand. Once a simulation is started, the host computer will not interfere with the operation of the simulation and the process of the simulation run entirely in accordance with the programs in the OCS.

\section{Loose Cluster Flight Demonstration}

Loose cluster flight, which is a common flight mode for fractionated spacecraft cluster, does not 
require spacecraft to maintain precision relative station-keeping, but only to keep the distance between two spacecrafts within a certain range to ensure that spacecrafts can communicate, and to ensure no collision risk between spacecrafts. On the simulation platform, a task simulation of loose flight formation including four spacecrafts is performed.

\subsection{Information Exchange Scheme}

The information exchanged between the on-board computer simulators includes the spacecraft ID, the reference spacecraft ID, control mark, time stamp, and the position and velocity of a spacecraft, illustrated in Fig. 6. The spacecraft ID is a unique identity number for each spacecraft, and is used to determine the information source. The reference spacecraft ID denotes whether the spacecraft is the center of the cluster and other spacecrafts in the cluster should flight referring to its orbit. The control mark illustrates the control state of a spacecraft. When the distance between two spacecrafts is close to be in collision risk or is too far to lose wireless communication, they will control themselves to change the distance. The time stamp records the running time from the simulation starting. The position and velocity of a spacecraft, corresponding to the time stamp, are acquired from the dynamical simulator.
Each spacecraft in the cluster broadcasts its information. Thus, each spacecraft can receive information of other spacecrafts within the wireless communication range. To simplify the demonstration, here the hypothesis that the four spacecrafts could always receive information from each other is adopted.

\subsection{Spacecraft Control Scheme}

The spacecraft cluster control scheme includes two rules: the reference spacecraft determination rule and the position-keeping rule. The reference spacecraft determination rule chose the spacecraft near to the center of the cluster to be the reference spacecraft. According to the position-keeping rule, other spacecrafts in the cluster can calculate their sequence in the cluster respectively. The detail of the rules was discussed in Ref. [14].

\subsection{Demonstration Scenario}

To validate the simulation system, a typical loose cluster flight demonstration was performed on that simulation platform. The cluster flight scenery begins at 12:00, June, 1st, 2008. And the initial orbit parameters of four spacecrafts are listed in Table 1.

Fig. 7 displayed the simulation results and it is obvious that the four spacecrafts formed a loose formation.

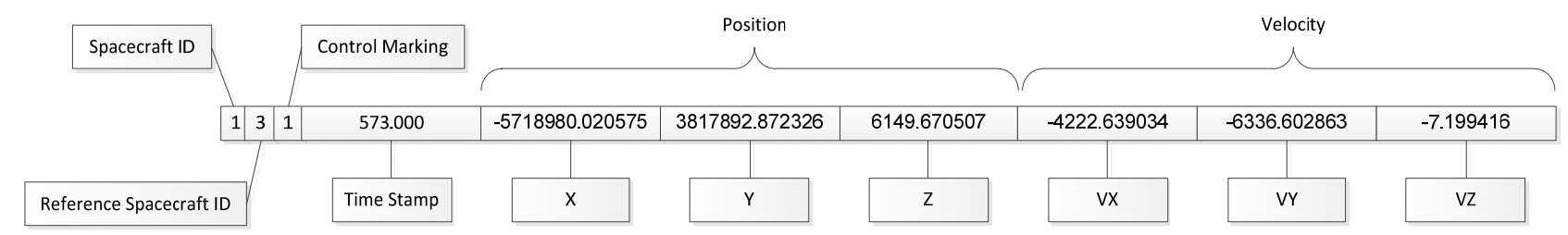

Fig. 6 Information exchanged between on-board computer simulators.

Table 1 Initial orbit parameters of the cluster.

\begin{tabular}{lcccc}
\hline Spacecraft ID & 1 & 2 & 3 & 4 \\
\hline Orbit altitude (km) & 600 & 610 & 620 & 630 \\
Eccentricity & 0 & 0 & 0 & 0 \\
Orbital inclination (degree) & 97.4825 & 97.4825 & 97.4825 & 97.4825 \\
Longitude of the ascending node (degree) & 9.7797 & 9.7787 & 9.7777 & 9.7767 \\
Argument of perigee (degree) & 0 & 0 & 0 & 0 \\
Mean anomaly (degree) & 100.1 & 100.2 & 100.3 & 100.4 \\
\hline
\end{tabular}




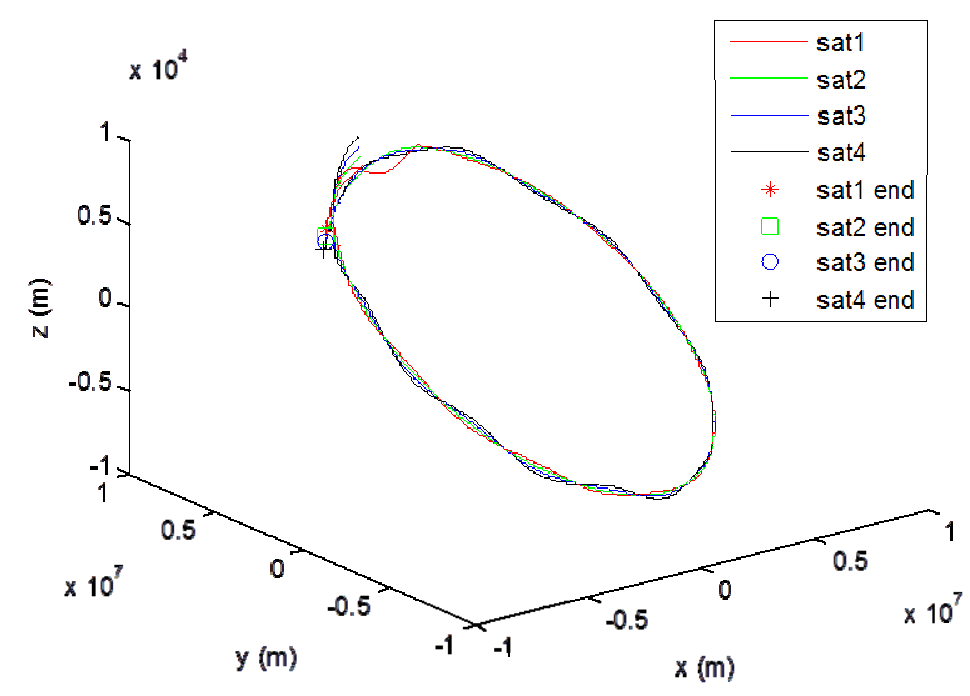

Fig. 7 Result of loose cluster flight demonstration.

\section{Conclusions}

A NNP for spaceborne ad-hoc network was built up with chip Jennic 5148 as its hardware. Based on four network node prototypes, a hardware-in-loop simulation system for FSC was established. A typical loose cluster flying mission, with distributed following-and-queuing-tactic adopted to manage each of the four spacecrafts, is performed in this simulation system. The simulation results demonstrate that the four spacecrafts form a loose formation. The performance of the system indicates that the network node prototype effectively supports FSC distributed control simulation. The hardware-in-loop simulation system establishes a control strategy testing platform, which could be utilized to evaluate the collective behavior of spacecraft cluster in the future.

\section{Acknowledgements}

This research was sponsored by 863 project of \#2012AA1206030102 named "Network and Control of Module Intelligent Agent”.

\section{References}

[1] O’Neill, M. G., and Weigel, A. L. 2011. “Assessing Fractionated Spacecraft Value Propositions for Earth Imaging Space Missions.” Journal of Spacecraft and Rockets 48 (6): 974-86. doi: 10.2514/1.50947.
[2] Mathieu, C. 2006. "Assessing the Fractionated Spacecraft Concept.” Master thesis, Massachusetts Institute of Approach.

[3] Lübberstedt, H., Koebel, D., Hansen, F., and Brauer, P. 2005. "MAGNAS-Magnetic Nanoprobe Swarm.” Acta Astronautica $56 \quad$ (1): 209-12. doi: 10.1016/j.actaastro.2004.09.030.

[4] Hinchey, M. G., Rash, J. L., Truszkowski, W. F., Rouff, C.A., and Sterritt, R. 2005. "Challenges of Developing New Classes of NASA Self-managing Missions.” In Prodeedings of the 11th International Conference on Parallel and Distributed Systems, 463-7.

[5] Truszkowski, W. F., Hinchey, M. G., Rash, J. L., and Rouff, C. A. 2006. "Autonomous and Autonomic Systems: A Paradigm for Future Space Exploration Missions.” IEEE Transactions on Systems, Man, and Cybernetics, Part C: Applications and Reviews 36 (3): 279-91. doi: 10.1109/TSMCC.2006.871600.

[6] Curtis, S. A., Rilee, M. L., Clark, P. E., and Marr, G. C. 2003. "Use of Swarm Intelligence in Spacecraft Constellations for the Resource Exploration of the Asteroid Belt." Presented at the 3rd International Workshop on Satellite Constellations and Formation Flying, Pisa, Italy.

[7] D’Arrigo, P., and Santandrea, S. 2006. "The APIES Mission to Explore the Asteroid Belt." Advances in Space Research $38 \quad$ (9): 2060-67. doi: 10.1016/j.asr.2006.09.017.

[8] Owen, B., and Paul, E. 2006. "Fractionated Space Architectures: A Vision for Responsive Apace.” Presented at the 4th Responsive Space Conference, Los Angeles, USA.

[9] Royer, E. M., and Toh, C. K. 1999. “A Review of Current 
Routing Protocols for Ad Hoc Mobile Wireless Networks.” Personal Communications 6 (2): 46-55. doi: 10.1.1.10.4428.

[10] Toh, C. K. K. 2001. Ad Hoc Wireless Networks: Protocols and Systems. London: Prentice Hall PTR.

[11] Dengiz, O., Konak, A., and Smith, A. E. 2011. "Connectivity Management in Mobile Ad Hoc Networks Using Particle Swarm Optimization.” Ad Hoc Networks 9 (7): 1312-26. doi: 10.1016/j.adhoc.2011.01.010.

[12] Prasad, D. K., and Murty, S. V. S. 2011. "Wireless Sensor Networks-A Potential Tool to Probe for Water on Moon.” Advances in Space Research 48 (3): 601-12. doi: 10.1016/j.asr.2011.04.004.

[13] Dubois, P., Botteron, C., Mitev, V., Menon, C., Farine P., Dainesi, P., Ionescu, A., and Shea, H. 2009. "Ad hoc Wireless Sensor Networks for Exploration of Solar-System Bodies.” Acta Astronautica 64 (5): 626-43. doi: 10.1016/j.actaastro.2008.11.012.

[14] Jiang, C., Wang, Z. K., and Zhang, Y. L. 2012. "Satellite Cluster Autonomic Coordination Control Strategy Based on Parallel System Theory.” Orbital Operation Mission Space Dynamic Workshop. Xi'an, China. 